\title{
Mauro Magnoni*
}

\section{The first diffusion of the Covid-19 outbreak in Northern Italy: an analysis based on a simplified version of the SIR model}

\author{
https://doi.org/10.1515/em-2020-0047
}

Received October 29, 2020; accepted March 10, 2021; published online March 25, 2021

\begin{abstract}
In this paper an analysis of the first diffusion of the Covid-19 outbreak occurred in late February 2020 in Northern Italy is presented. In order to study the time evolution of the epidemic it was decided to analyze in particular as the most relevant variable the number of hospitalized people, considered as the less biased proxy of the real number of infected people. An approximate solution of the infected equation was found from a simplified version of the SIR model. This solution was used as a tool for the calculation of the basic reproduction number $R_{0}$ in the early phase of the epidemic for the most affected Northern Italian regions (Piedmont, Lombardy, Veneto and Emilia), giving values of $R_{0}$ ranging from 2.2 to 3.1. Finally, a theoretical formulation of the infection rate is proposed, introducing a new parameter, the infection length, characteristic of the disease.
\end{abstract}

Keywords: approximate solution; infectious lenght; SIR model.

\section{Introduction}

A sudden increase of cases of Covid-19 diseases originated by the new coronavirus SARS-CoV-2 struck Northern Italy and Lombardy in particular,inlate February 2020 (Distante et al. 2020; Italian National Institute of Health (ISS); Italian Ministry of Health). The rapid growth of many severe illnesses leads to a dramatic pressure on the hospitals, particularly in the intensive care units. In a few weeks thousands of people were hospitalized and roughly an equal number of persons found positive to the tests and with only mild symptoms, were quarantined at home. However, this was just the tip of the iceberg: the estimated incubation period of about 5 days but with a quite large range (2-14 days) (Lauer et al. 2020) clearly promotes the diffusion of the outbreak and, as a consequence of that, it was very soon clear that also mild, asymptomatic and pre-symptomatic cases played a significant role in the rapid surge of the epidemic (Li et al.). This fact was confirmed by many other studies (Gandhi et al. 2020) where also negative serial intervals $\mathrm{SI}^{1}$ have been reported (Du et al. 2020). Unfortunately, the lack of a quick availability of a large number of test kits prevented to know the real dimension of the outbreak diffusion. For that reason, the reliability of the official data given by the authorities was questioned: the number of people found positive was of course greatly influenced by the number of tests performed and, more importantly, the criteria followed to submit people to the tests, especially in the first weeks after the beginning of the outbreak, were not completely consistent in space and time. It is thus certain that the number of the infected people officially declared doesn't reflect the real number of infected individuals in the population.

Nevertheless, these limitations don't prevent the possibility of using the official data for some analyses. The most interesting features of the dynamics of an outbreak can be investigated studying the variations of the relevant variables, irrespective of their precise and "real" values.

1 The serial interval SI is the time between a primary case-patient (infector) and a secondary case-patient (infectee).

* Corresponding author: Mauro Magnoni, ARPA Piemonte, Department of Physical and Technological Risks, Via Jervis, 30, 10015 Ivrea (TO), Italy, E-mail: m.magnoni@arpa.piemonte.it 


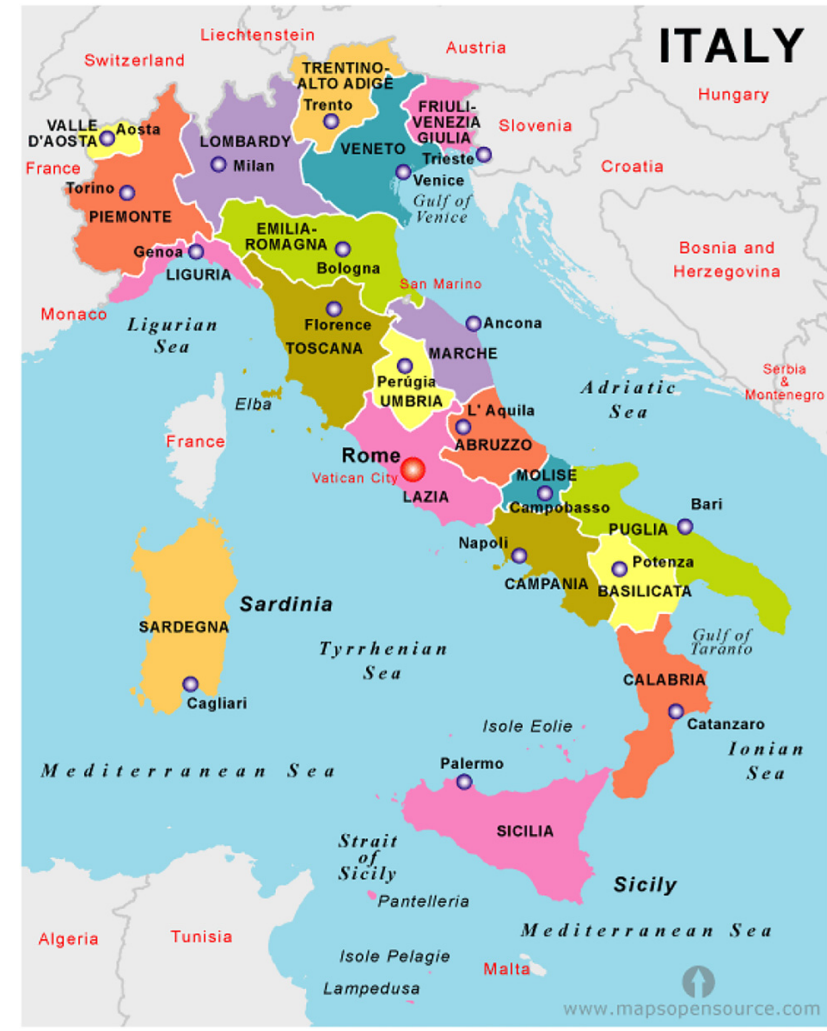

Figure 1: The Northern regions of Italy most affected by the Covid-19 outbreak: Piedmont, Lombardy, Emilia-Romagna and Veneto.

The official data were given on a daily basis and for each Italian region by the (Italian Department of Civil Protection). In this study the following data were considered: the number of infected people, unfortunately greatly affected by the number of tests performed, the number of hospitalized people and the number of people admitted in the intensive care units.

In order to study the time evolution of the epidemic we decided to use as relevant variable, besides the number of infected people, also the number of the hospitalized, considered as a less biased proxy of the real number of infected people, the latter being potentially influenced by the unknown number of undetected cases. The rationale of this choice is that the number of hospitalized patients depends essentially on the epidemic dynamics and should not be greatly affected by some "external factors" such as, for example, the varying availability of tests and the changing of policies of the health authorities during the emergency. In doing so, there are of course some very important underlying assumptions, the most important one is that the susceptibility to the disease within the affected population is assumed consistent in space and time. This condition is certainly not completely true, as it is well known that the susceptibility of a given population to this disease is largely influenced by its age structure and density. A correction for these effects are possible in principle, provided that detailed information on the age structure of the populations and their densities are taken into account. However, it was decided to not include these aspects in this preliminary work: the data were taken as they are and the results were discussed. Nevertheless, no significant biases are expected due to the lack of consideration of the population ages structure because their distributions are quite similar within the considered Italian regions. All data are interpreted in the light of the SIR model (Kermack and McKendrick 1927), i.e. the standard model describing the evolution of the outbreaks in term of susceptible people (S), the infected (I) and the recovered (R) ones, presented here in an approximate and simplified form.

As the Covid-19 epidemic affects Italy very unevenly, with the Northern regions much more affected than the rest of the country, this work is mainly focused on the most affected ones, in particular: Lombardy, Piedmont, Emilia-Romagna and Veneto (Figure 1). Sicily was also considered for the sake of comparison, as a typical representative of the Southern regions. 


\section{Theoretical background}

The standard SIR model, first proposed in 1927 by Kermack and McKendric (Kermack and McKendrick 1927), is a model described by a system of three non linear differential equation connecting three compartments representing the stocks of the susceptible population (S), the infected (I) and the recovered (R) ones.

The mathematical formulation of the model is shown in the following, in which $x(t)$ represents the susceptible population, $y(t)$ the infected, $z(t)$ the recovered, while the parameters $\beta$ and $\gamma$ are respectively the infection rate and the recovery rate $^{2}$ :

$$
\begin{gathered}
\frac{\mathrm{d} x(t)}{\mathrm{d} t}=-\frac{\beta}{N} \cdot x(t) \cdot y(t) \\
\frac{\mathrm{d} y(t)}{\mathrm{d} t}=\frac{\beta}{N} \cdot x(t) \cdot y(t)-\gamma \cdot y(t) \\
\frac{\mathrm{d} z(t)}{\mathrm{d} t}=\gamma \cdot y(t)
\end{gathered}
$$

In these equations $N$ is a constant, representing the total number of people that can be potentially involved (fixed population). The boundary conditions of the system are generically given by: $x(0)=N_{1} ; y(0)=$ $N_{2} ; z(0)=N_{3}$, with the following constraint, $N=N_{1}+N_{2}+N_{3}$. The dynamics of the infection is governed by the ratio $R_{0}=\beta / \gamma$, the so called basic reproduction number, the parameter usually utilized for the description of the diffusion of an epidemic: the greater is $R_{0}$, the greater and faster is the spread of the outbreak, while a value of $R_{0}<1$ leads to the extinction of the disease.

However, while $\gamma$ is a constant depending essentially on the medical characteristics of the disease and, for that reason, can't be substantially affected by actions taken during an epidemic, the reduction of the $R_{0}$ value can be achieved only by lowering the infection rate $\beta$, usually done introducing measures such as social distancing. This kind of measures were actually taken by the Italian government, in particular from the 8th March (DPCM 2020): a strict lockdown was imposed all over Italy in the effort to reduce the spread of the disease in the whole country.

Going back to the theoretical framework, it can be observed that the solutions of the equations system are usually performed numerically using different mathematical approaches (Biazar 2006; Rafei et al. 2007a, 2007b). An exact analytical solution of the SIR equations was quite recently (2014) proposed in a parametric form (Harko et al. 2012). However, all these methods are not easy to be handled and require some technicalities not always easily available among many researchers working in the medical field. For those reasons in this work we will derive an approximate analytical solution of the system, giving very simple formulas much easier to be used and applied to real conditions.

The basic assumption we made was substituting at the right side of the first Eq. (1a), the time varying function $y(t)$, representing the infected population with its average value $\bar{y}$, calculated in the time range $\Delta \tau$ during which the outbreak develops, namely:

$$
\bar{y}=\frac{1}{\Delta \tau} \cdot \int_{0}^{\Delta \tau} y(t) \mathrm{d} t
$$

The first Eq. (1a) can thus be rewritten in the following form:

$$
\frac{\mathrm{d} x(t)}{\mathrm{d} t}=-\frac{\beta}{N} \cdot x(t) \cdot \bar{y}
$$

that is straightforward integrated giving the following expression:

$$
x(t)=x_{0} \cdot \mathrm{e}^{-\frac{\beta}{N} \cdot \bar{y} \cdot t}
$$

2 It is worth to note that in this model the $\gamma$ parameter in the equation represents more precisely the rate of removal from the infected compartment rather than a real recovery rate. Actually, a complete recovery in a clinical sense may take more time and does not coincide with the time lag during which the infected patient is capable to spread the disease. 
in which $x_{0}$ is the susceptible population stock at time zero. The second equation, representing the stock of the infected population, thus becomes:

$$
\frac{\mathrm{d} y(t)}{\mathrm{d} t}=\frac{\beta}{N} \cdot x_{0} \cdot \mathrm{e}^{-\frac{\beta}{N} \cdot \bar{y} \cdot t} \cdot y(t)-\gamma \cdot y(t)
$$

This equation can be easily integrated by separation of the variables. We have therefore the general solution:

$$
\ln y(t)=-\frac{x_{0}}{\bar{y}} \cdot \mathrm{e}^{-\frac{\beta}{N} \cdot \bar{y} \cdot t}-\gamma \cdot t+C
$$

where $C$ in an arbitrary integration constant whose value is determined by the boundary condition $y(0)=y_{0}$, where $y_{0}$ is a non zero value representing the number of infected people at time $t=0$, i.e., at the starting of the outbreak. Therefore, we have:

$$
\ln y_{0}=-\frac{x_{0}}{\bar{y}}+C
$$

from which the value of the integration constant can be calculated, giving:

$$
y(t)=y_{0} \cdot \mathrm{e}^{\frac{x_{0}}{\bar{y}}\left(1-\mathrm{e}^{-\frac{\beta}{N} \cdot \bar{y} \cdot t}\right)} \cdot \mathrm{e}^{-\gamma \cdot t}
$$

that is the general solution of the equation with the given boundary conditions. A very interesting approximate form of this expression can be found for small $t$ values, i.e. for the early days of the outbreak. Expanding at the first order the term $\mathrm{e}^{-\frac{\beta}{N} \cdot \bar{y} \cdot t}$ in the exponent we can write:

$$
y(t)=y_{0} \cdot \mathrm{e}^{x_{0} \cdot \frac{\beta}{N} \cdot t-\gamma \cdot t}
$$

Making then the very reasonable assumption that $x_{0} \approx N$ and remembering that by definition we have $R_{0}=\beta / \gamma$, the above equation can be put in the following form:

$$
y(t)=y_{0} \cdot \mathrm{e}^{\gamma \cdot\left(R_{0}-1\right) \cdot t}
$$

This expression shows the well known condition for the epidemic diffusion (exponential growth only if $R_{0}>1$ ) and also provides a simple tool for the calculation of the $R_{0}$ parameter in the early phase of the outbreak.

Besides this, another interesting theoretical relationship involving the basic reproduction number $R_{0}$ can be derived. Being $R_{0}=\beta / \gamma$, where the parameters $\beta$ and $\gamma$ are respectively the infection and the recovery rate, it is clear that the dependence from the environmental conditions is described by the factor $\beta$. Indeed, it is obvious that the infection rate $\beta$ observed in a population depends on the probability of occurrence of close contacts between the individuals of the population itself. Moreover it seems reasonable to assume that this probability will be proportional to the velocity $v$ of the movements of the population: the greater the velocity of the displacement of an individual, the larger the chance to meet another person. Therefore, for a given population of size $N$ and living in an area whose extension is $S$, the infection rate can be expressed as follows:

$$
\beta=v \cdot \frac{N}{S} \cdot \ell
$$

where $v$ is the mean displacement velocity of the individuals of the population, $N$ is the number of the individuals of the population, $S$ is the area in which the population lives and $\ell$ is a key parameter, expressing the contagiousness of the disease (whose dimension is a length). It could be named as the "infection length", being interpreted as the mean distance for an effective infectious contact. It is worth noting that in this description the parameter $\ell$ plays a role very similar to that of the cross section in nuclear interactions (Alonso and Finn 1983). Following this theoretical framework allows to distinguish in the infection rate $\beta$, two different factors: a population factor, $v \cdot \frac{N}{S}$, depending on the density of the populations and on its habits, and a biological factor $\ell$, mainly depending on the characteristic of the virus and possibly also on some environmental "external" variables such as, for example, the temperature and the humidity. The measures taken by the authorities can act on both factors: in particular, the general lockdown imposed to all the country 
from the 8th March (DPCM 2020), reducing the movements of the persons, lowered dramatically the $v$ value, while the obligation for all the population of wearing masks obviously reduced the $\ell$ value. These measures were proved to be quite effective, leading to a rapid decrease of the $R_{0}$ value below 1 , thus stopping the diffusion of the outbreak.

\section{Data analyses, results and discussion}

The official data of the Covid-19 outbreak in Italy were given by the authorities (Italian Department of Civil Protection) from the 24th February 2020 and, from that moment, updated daily (Italian Department of Civil Protection). The analyses of the data made in this work are performed on a regional basis rather than at a national level because the regional data are more homogeneous and there were significant temporal shifts (Pagano et al. 2020) in the development of the outbreak in the different Italian regions. We focused mainly on the most affected Regions, located in the North of the country such as: Lombardy, Piedmont, Veneto, Emilia-Romagna while, for the sake of comparison, also the data from Sicily are also considered.

In Figures 2-4 the temporal evolution of the outbreak is reported for Lombardy, Piedmont, Veneto, and Emilia-Romagna by means of three different variables: the number of total infected people (Figure 2), the number of Covid-19 hospitalized patients (Figure 3) and the number of Covid-19 occupied intensive care units (Figure 4).

It appears at first sight that the curves representing the number of total infected people (Figure 2), in particular for Lombardy and Piedmont, look different from the others (Figures 3 and 4): they are less smooth and, more interestingly, the maximum is much broader and shifted forward by about 30 days. Actually, the maximum of the curves in Figures 3 and 4 occurs around the first days of April while for Figure 2 it occurs about one month later. This fact is not surprising, as the number of daily tests performed in both Regions increased from April to May approximately 5 times, thus changing dramatically the capability of detection of the new cases, causing the observed increase of the number infected people. Therefore, the global trend of the "total infected" curve is severe biased and should not be considered as a reliable description of the real evolution of the outbreak. A more reliable picture of the situation can be obtained observing the curves of the hospitalized patients and those representing the number of occupied intensive care units as well. They both look smoother and, more importantly, for the reason already discussed in the introduction, they do not suffer the distortions due to the day by day changing number of the tests performed. Therefore, assuming that the patients needing hospitalization is a fixed fraction of the total infected population, these curves (Figures 3 and 4) show the "real" evolution of the outbreak, at least in the first weeks.

Investigating the early stage of the epidemic, in a time range spanning the first 18 days, from the 24th February to the 13th March, it can be seen that the growth of the number of infected people follows approximately an exponential law with very short doubling time, ranging from 2 to 3.6 days. Afterwards the growth deviated substantially from an exponential law, almost certainly as consequence of the strict

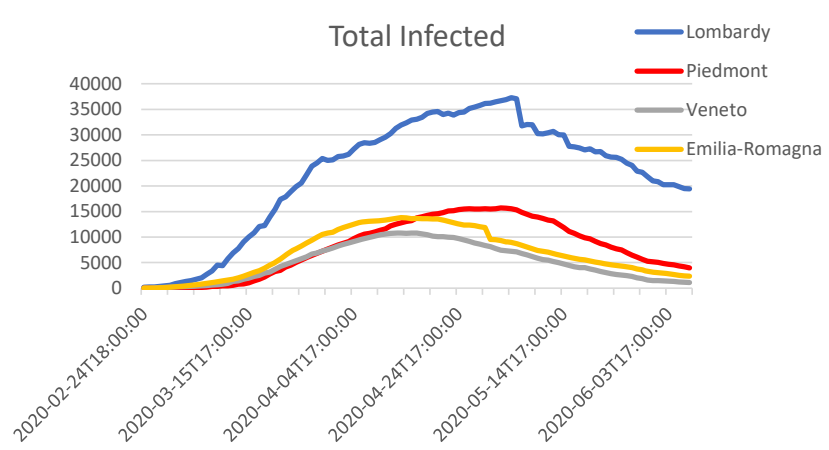

Figure 2: Number of the total infected people in Lombardy, Piedmont, Veneto and Emilia-Romagna. 

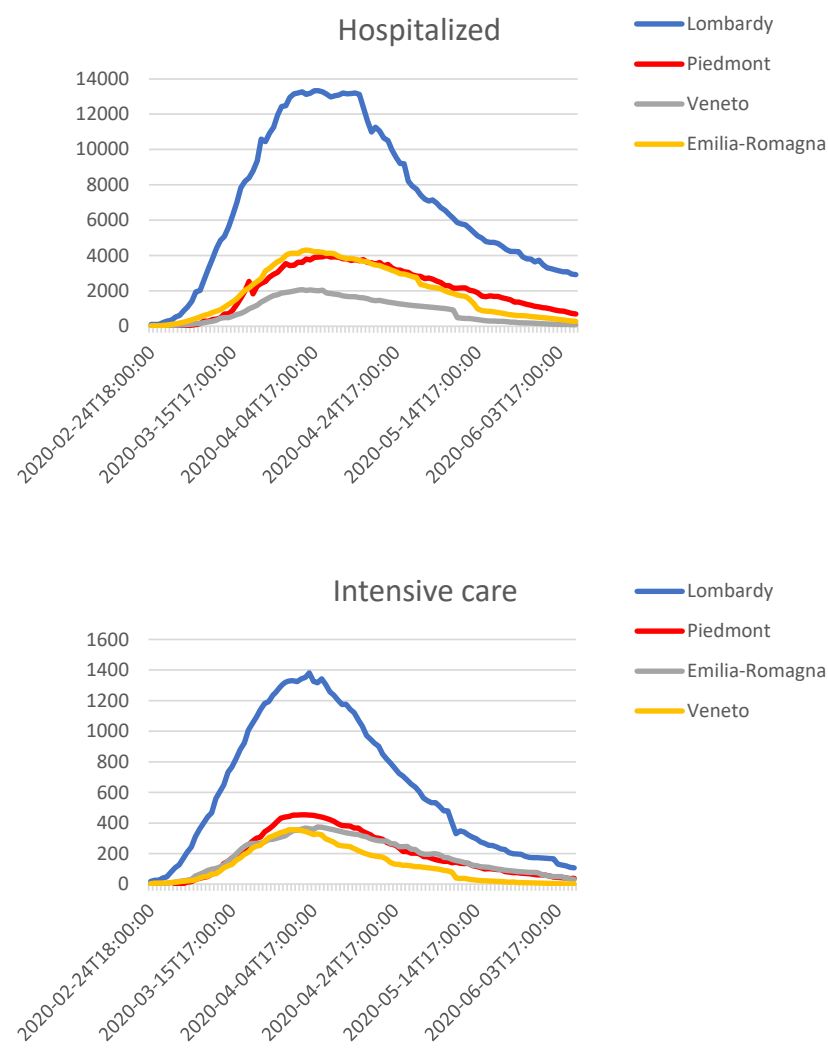

Figure 3: Number of hospitalized people in Lombardy, Piedmont, Veneto and Emilia-Romagna.

Figure 4: Number of people in intensive care units in Lombardy, Piedmont, Veneto and Emilia-Romagna.

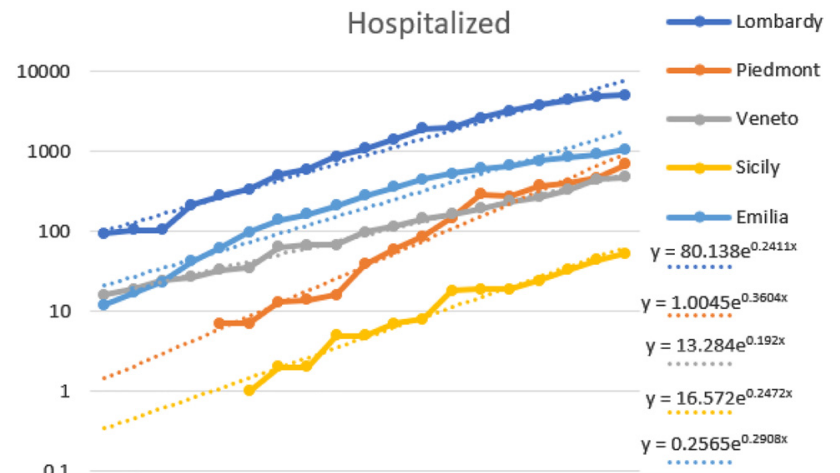

Figure 5: Exponential growth in some Northern Italian regions plus Sicily (hospitalized).

lockdown firstly enforced by the Italian Government in the most affected regions (2020 (Lombardy and a large part of the territories of Emilia-Romagna, Veneto and Piedmont) from 8th March and soon after, from 10th March in the whole country (DPCM 2020): these measures were taken in the effort to stop the exponential growth and to limit the spread of the outbreak to the Southern regions.

In Figures 5 and 6 the data referred to the hospitalized patients and the total infected ones for five regions: Lombardy, Piedmont, Emilia-Romagna, Veneto and Sicily, are shown with their corresponding interpolation exponential fit functions, namely: $y(t)=y_{0} \cdot \mathrm{e}^{r \cdot t}$ in which $y_{0}$ and $r$ are coefficients to be determined fitting the experimental data.

These data can be used to evaluate the reproduction number $R_{0}$ at the beginning of the epidemic. Actually, from Eq. (3) the following expression can be derived:

$$
R_{0}=\frac{r}{\gamma}+1
$$




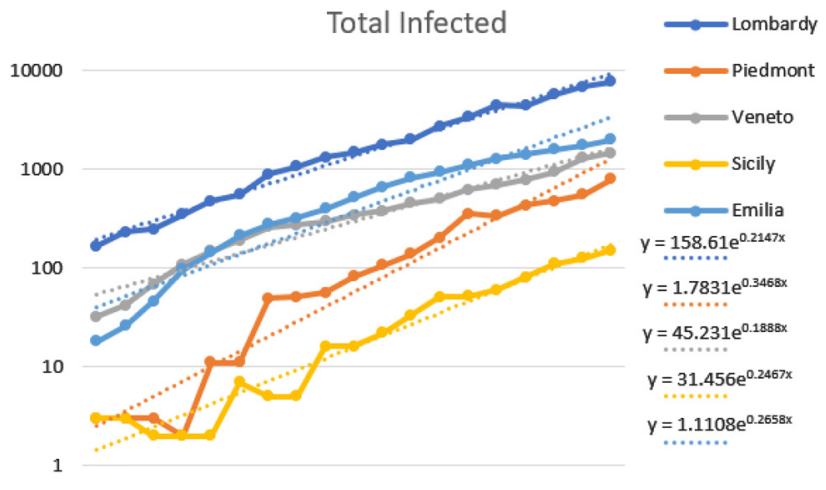

Figure 6: Exponential growth in some Northern Italian regions plus Sicily (total infected).

in which $R_{0}$ is related to the observed growth rate $r$ and the recovery rate $\gamma$, which is the reciprocal of the average infectious period IP, i.e. the average time interval during which an infected individual is capable to spread the disease. Inferring a reliable IP value for Covid-19 is not a simple task at the moment, due to the large heterogeneity of the available data. Many different approaches were proposed in order to evaluate a proper IP value, by means of virological, contact tracing and modelling studies (Byrne et al. 2020). For example, Ma et al. (Ma et al. 2020) estimated a mean IP value of 7.25 days as the difference between the upper latent period minus the average serial interval SI, Li et al. gave a median value of 3.5 days (estimated mean 4.35) (Li et al. 2020); Tuite et al. found a mean value of 6.25 days (Tuite et al. 2020); Davies et al., in two different modelling studies (Davies et al. 2020a, 2020b) found averages values respectively of 5 and 7 days. Considering all these data and assuming that a triangular distribution holds between the minimum and the maximum of these numbers, a representative average value and the standard deviation of the infectious period IP can be computed, giving IP $=5.90 \pm 0.59$ days, corresponding to $\gamma=0.17 \pm 0.02$ days $^{-1}$. This range of values can thus be considered as the input data for the calculation of the $R_{0}$ values for all the regions accordingly with the expression reported in Eq. (5) in which a key role is played by the growth rate $r$ estimated interpolating the experimental data (Figures 5 and 6), representing respectively the number of hospitalized and the number of the total infected people. The curves look quite similar for every region, as can be seen also in Table 1, where the values of the coefficients $y_{0}$ and $r$ of the interpolating exponential functions are displayed and shown together with their corresponding $R_{0}$ values (mean values and ranges, $90 \%$ IC).

The initial growth rate $r$ in the different regions ranges from 0.19 days $^{-1}$ (Veneto) to 0.36 days $^{-1}$ (Piedmont), the average being 0.27 days $^{-1}$, close the value found by Gaeta $(r=0.32)$ (Gaeta 2020), in an early work limited to Veneto, Emilia-Romagna and Lombardy. The mean values of the obtained $R_{0}$ span an interval from a minimum of 2.11 (Veneto) to a maximum of 3.12 (Piedmont), the whole average being $2.53 \pm 0.35$. These estimates are consistent with those calculated in Wuhan, $\left(R_{0}=2.68\right)$ (Zhou et al. 2020; Zhao et al. 2020; TWu et al. 2020) and lower than those given by Distante et al. for Italy, estimated in the early phase of the outbreak, following a different approach (Distante et al. 2020). Little greater $R_{0}$ values than those obtained

Table 1: Values of the interpolating coefficients of the curves (initial value $y_{0}$ and growth rate $r$ ) with the corresponding $R_{0}$ estimates.

\begin{tabular}{|c|c|c|c|c|c|c|c|c|}
\hline \multirow[t]{2}{*}{ Region } & \multicolumn{4}{|c|}{ Hospitalized } & \multicolumn{4}{|c|}{ Total infected } \\
\hline & $y_{0}$ & $r\left(\right.$ days $\left.^{-1}\right)$ & $R_{0}$ mean & $R_{0}$ range & $y_{0}$ & $r\left(\right.$ days $\left.^{-1}\right)$ & $R_{0}$ mean & $R_{0}$ range \\
\hline Lombardy & 80.14 & 0.24 & 2.42 & $2.27-2.61$ & 158.61 & 0.21 & 2.26 & $2.13-2.43$ \\
\hline Piedmont & 1.01 & 0.36 & 3.12 & $2.90-3.40$ & 1.78 & 0.35 & 3.04 & $2.83-3.31$ \\
\hline Emilia-Romagna & 16.57 & 0.25 & 2.45 & $2.30-2.65$ & 31.46 & 0.25 & 2.45 & $2.30-2.64$ \\
\hline Veneto & 13.28 & 0.19 & 2.13 & $2.01-2.28$ & 45.23 & 0.19 & 2.11 & $1.99-2.26$ \\
\hline Sicily & 0.26 & 0.29 & 2.71 & $2.53-2.94$ & 1.11 & 0.27 & 2.56 & $2.40-2.77$ \\
\hline
\end{tabular}


with our method can be found (for example $R_{0}=2.88$ for Lombardy) analyzing our data by means of a widely used software package (Cori et al. 2013) implementing a numerical solution of the SIR model for the calculation of the time-varying reproduction number.

Comparing the two datasets, we can observe that the "hospitalized" data show values a little bit larger than the "total infected" ones, while the differences are quite small: it seems that the diffusion of the outbreak, at least in the early phase, could be suitable described by either variables, the "hospitalized" and the "total infected" as well. For some regions, Lombardy in particular ( $R_{0}$ averages, respectively 2.42 and 2.26 ), but to some extent also for Veneto ( $R_{0}$ averages, respectively 2.13 and 2.11) and Emilia-Romagna (same $R_{0}$ averages, 2.45, for both "hospitalized" and "total infected"), the available data from which the values are derived, starting from the 24th February, missed the real beginning of the epidemic. Actually, a simple extrapolation made by using Eq. (3) dates back the starting of the outbreak at least by about 18 days for Lombardy, 13 days for Veneto and 11 days for Emilia-Romagna. This figure is consistent with some recent evaluations showing evidence of the circulation of the virus in Northern Italy well before the official dates (Goumenou et al. 2020). As a consequence of that, for these three regions the calculated $R_{0}$ values could be slightly underestimated as the mathematical approximations made for the derivation of Eq. (3) do not precisely hold after about 25-30 days from the beginning of the outbreak. This fact could also explain the observed higher values found for Piedmont $\left(R_{0}=3.12\right)$ and Sicily $\left(R_{0}=2.71\right)$ where the outbreak developed a few days later, allowing thus a more accurate experimental observation of the initial growth rate. However, from these data it seems clear that the dynamics of the outbreak doesn't differ significantly from one region to another: the initial exponential growths are quite similar in most regions. A partial exception to this figure may be represented by Veneto, which shows a little lower $R_{0}$ value that couldn't be due only by the missing data of the beginning of the outbreak. In this case it seems reasonable to argue that a significant role was also played by some other factors such as: (a) the more strict measures taken by the local health authorities of the Veneto region: the people of first infected villages were immediately quarantined and a quite larger number of tests, compared to other regions, were performed on the population in order to a prompt identification of the infected individuals; (b) most of the towns involved in the first contagion episodes were quite small, characterized by a lower population density ${ }^{3}$. For example, the population of Vo' Euganeo, a little village in Veneto (about 3,300 inhabitants), one of the first struck by the outbreak, it has a population density of 161.86 inhabitants $/ \mathrm{km}^{2}$, significantly lower, for example, than those of the Lombard towns and villages in the Bergamo Province (the most affected Covid-19 Italian area), where the population densities are up to one order of magnitude greater (Italia in cifre 2013).

After the initial exponential growth, all the curves of the infected population (Figures 3-5) exhibit a very similar behavior: a progressive decreasing growth rate resulting in a broad maximum about 50 days after the beginning, followed by a slow decrease. In our theoretical description this behavior is modelled with Eq. (2). Considering the curves of the hospitalized people (Figure 4) as representative for the infection time evolution, a $\chi^{2}$ fit was performed for each curve with two free independent parameters to be determined, $a$ and $b$, as follows:

$$
\chi(a, b)^{2}=\sum_{j=1}^{m}\left(y_{o} \cdot \mathrm{e}^{b \cdot\left(1-\mathrm{e}^{-a \cdot j}\right)} \cdot \mathrm{e}^{-\gamma \cdot t}-\operatorname{Hosp}_{j}\right)^{2}
$$

where $\mathrm{Hosp}_{j}$ represents the number of hospitalized patients in the day $j$. The results of these calculations are shown in the following Figure 7(a)-(e), for all the considered regions.

The agreement of the fit functions with the experimental data is qualitatively good while in some cases significant deviations between the theoretical curves and the experimental data appear. In particular, after reaching the maximum, the real values decrease much more slowly than the theoretical ones: this happens in particular for the Lombard and Piedmont data. A substantial improvement of the agreement can be obtained

3 This fact may of course influence the $R_{0}$ value. Indeed, being by definition $R_{0}=\beta / \gamma$ and taking into account for Eq. (4), we can write: $R_{0}=\frac{v}{\gamma} \cdot \frac{N}{S} \cdot \ell$. 
leaving free in the fitting calculation a third parameter, the recovery rate $\gamma$ (Eq. (6)). The results are shown in the following Figure 8(a) and (b).

The $\gamma$ values obtained with the fitting procedures are significantly lower than how previously assumed $\left(\gamma=0.17\right.$ days $\left.^{-1}\right)$ following the literature data. Actually we have found: $\gamma=0.062$ and $\gamma=0.071$ days $^{-1}$ for Lombardy and Piedmont respectively. These numbers would correspond to an average infectious period of
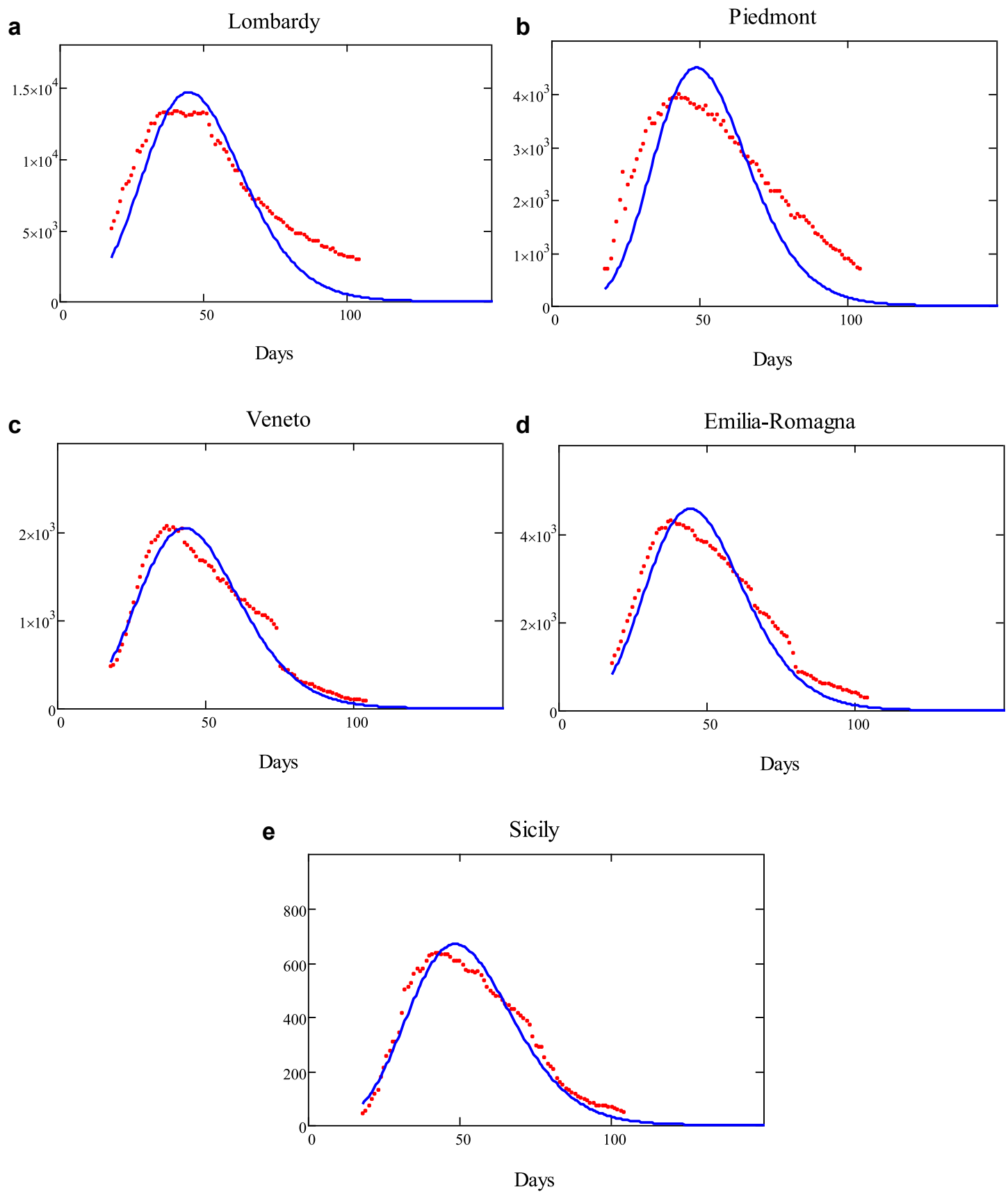

Figure 7: (a) Dynamics of the outbreak in Lombardy: experimental data (red points) and theoretical description (blue curve). (b) Dynamics of the outbreak in Piedmont: experimental data (red points) and theoretical description (blue curve). (c) Dynamics of the outbreak in Veneto: experimental data (red points) and theoretical description (blue curve). (d) Dynamics of the outbreak in Emilia-Romagna: experimental data (red points) and theoretical description (blue curve). (e) Dynamics of the outbreak in Sicily: experimental data (red points) and theoretical description (blue). 
about 14-16 days, certainly too large values to be considered reliable. However, this apparent inconsistency can be easily explained considering that the fitted experimental data refer to the hospitalized people, not to the infected ones: thus the 14-16 days calculated as the reciprocal of the $\gamma$ fitted values must be interpreted as the average time needed for a complete clinical recovery rather than the average infectious period IP (see note number 2).

Let's finally conclude the discussion with some considerations on the infection rate Eq. (4) written above. From this formula, taking into account that by definition we have $R_{0}=\beta / \gamma$, an interesting expression for the "infection length" parameter $\ell$ can be derived:

$$
\ell=\frac{\gamma}{v} \cdot \frac{R_{0}}{N / S}
$$

in which the dependence of this quantity from 4 different variables is shown: the recovery rate $\gamma$, the mean population displacement velocity $v$, the reproduction number $R_{0}$ and the population density $\frac{N}{S}$. A detailed discussion about the proper values to be attributed to these parameters is beyond the scope of this paper. The core of the question is the product $v \cdot \frac{N}{S}$, that should be considered as a unique variable, representing the number per interactions per unit time and length. Nevertheless, some heuristic consideration can be drawn now, considering separately all the variables. The order of magnitude of the population density in the considered regions ranges approximately from 193 to 423 inhabitants/km2 (ISTAT, (Italia in cifre 2013)). However, these are averages that do not represent the real condition experienced by most of the Italian population that lives in urban areas where the densities are much greater. For that reason it seems more appropriate for each region to consider $\rho$, defined as the weighted mean of the population densities over all the municipalities of the region, as follows:

$$
\rho=\sum_{J=1}^{m} w_{j} \cdot\left(\frac{N}{S}\right)_{j}
$$

where the $\left(\frac{N}{S}\right)_{j}$ is population density of the $j$ th municipality, $w_{j}=P_{j} / P_{\text {tot }}$ is the corresponding weight, being $P_{j}$ the population of the $j$ th municipality and $P_{\text {tot }}$ the total population of the region. The calculation of $\rho$ gives quite greater values: for example we have for Piedmont $\rho=1778$ inhabitants $/ \mathrm{km}^{2}$ and for Lombardy $\rho=2,241$ inhabitants $/ \mathrm{km}^{2}$. Thus, it can be assumed that the order of magnitude of this parameter should be $\rho=2000$ inhabitants $/ \mathrm{km}^{2}$.

Much more difficult is to estimate a reliable value for the mean population velocity $v$. This parameter can be interpreted as an effective velocity, not a real one, being obtained averaging all the displacement velocities
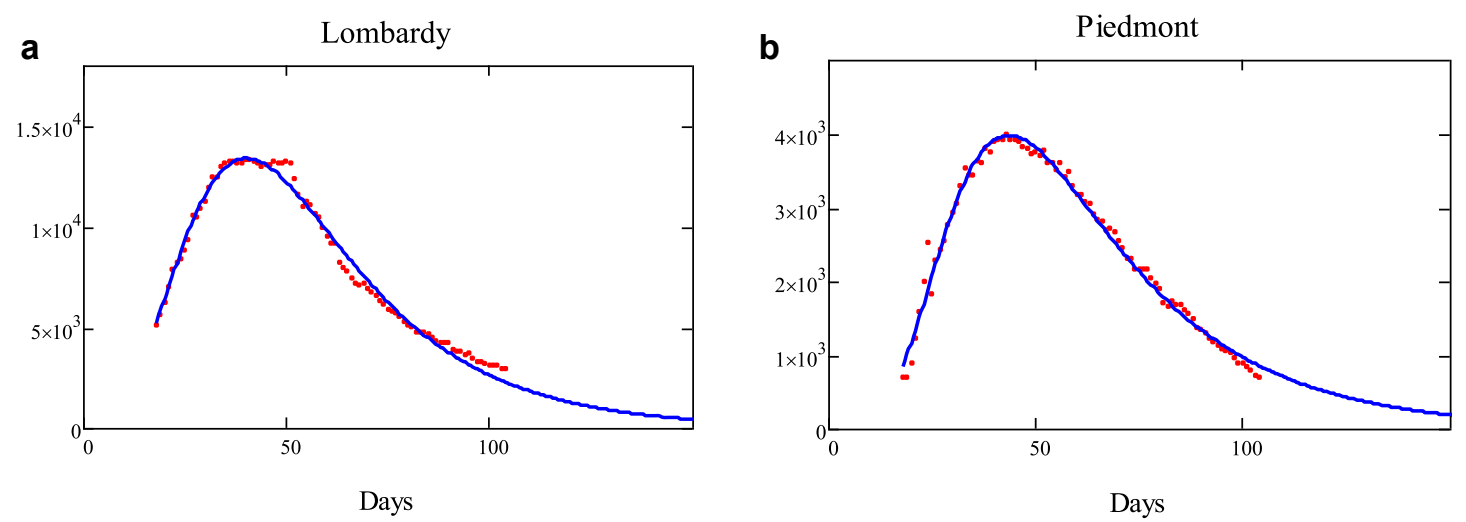

Figure 8: (a) Dynamics of the outbreak in Lombardy: Theoretical description (blue curve) with 3 free fitting parameters: the agreement between the curve and the data improves substantially (see Figure 7(a)). (b) Dynamics of the outbreak in Piedmont: theoretical description (blue curve) with 3 free fitting parameters: The agreement between the curve and the data improves substantially (see Figure 7(b)). 
over the whole population. In order to find a meaningful value for $v$ the following reasoning can be made. A normal walking speed for an adult ranges from 3.6 to $5.8 \mathrm{~km} / \mathrm{h}$ (Harkema et al. 2012). However, considering that a person doesn't walk continuously all the day, it seems reasonable to estimate the mean displacement velocity averaging over the whole day. Therefore, the effective average daily velocity is much smaller than the real values, resulting in about $v=0.04 \mathrm{~km} / \mathrm{h}$ (Statista 2020). Thus, if we take for $R_{0}$ the values experimentally obtained (spanning the range 2.01-3.40) , $^{4}$ it is possible to calculate the order of magnitude of the "infection length" $\ell$, defined above. Putting these numbers into Eq. (5) we find: $\ell \approx 18-30 \mathrm{~cm}$, that can be considered as the order of magnitude of the distance between individuals for an effective contagiousness for the Covid-19. This seems a very reasonable value, since accordingly with many studies (Jones et al. 2020) this distance was estimated being less than $1 \mathrm{~m}$.

\section{Conclusions}

The development of the Covid-19 outbreak in Northern Italy started in late February 2020 and showed a very similar behavior in all the main affected regions: a very fast exponential growth was observed in the first two weeks from the beginning of the epidemic. No significant differences appear among the different regions: the very different number of affected people between some regions seems simply due to the different initial conditions experienced in each region rather than from some supposed different environmental characteristics as claimed in some early investigations (Becchetti et al. 2020; Coker et al. 2020).

The analysis of the dynamics of the epidemic was performed considering as the most relevant variable, particularly in early phase of the epidemic, the number of hospitalized people, being the total number of infected severely biased by the varying and not homogeneous criteria followed in the testing procedures and also influenced by the unknown number of undetected cases. The available data were interpreted using a simplified formulation of the SIR model, allowing the calculation of the reproduction number $R_{0}$, the most relevant parameter of the outbreak's dynamics, by means of a simple linear regression analysis of the logaritms of the raw data. The global average value for all the considered regions was estimated as $R_{0}=2.53 \pm 0.35$, in good agreement with most of the available data. The method used also permitted to find an approximate solution for the infected Eq. (2) that fitted quite well with the experimental figures; actually, the deviations from the experimental data observed in some cases (on the tails of the curves, see Figure 7) seems due the fact that the variable used (number of hospitalized people) in the long range overestimate the real number of infected individuals.

However the proposed method, notwithstanding these limitations, introducing a formula giving a new global, albeit approximate, analytical solution of the SIR equations, was able not only to allow a very easy $R_{0}$ evaluation in the early phase of the outbreak without the need of any kind of software package, but also to give a good description of the experimental data even in the long run (see Figure 8).

Given the encouraging results obtained, the use of this simple mathematical formulation to other data sets (from Italy and from other countries as well) can be recommended and is currently on the way in order to test the performance of this method also in different contexts.

Finally, a general formulation for the infection rate $\beta$ was given, introducing and calculating the "infection length" $\ell$, a Covid-19 characteristic parameter. In general, this quantity should be considered as the key parameter for assessing the contagiousness of any specific contagious disease. In fact, given the $\ell$ value, the proposed theoretical approach (see Eq. (5)) could allow in principle the calculation of the basic reproduction rate $R_{0}$ simply from some "population data": the population density $\frac{N}{S}$ and the mean displacement velocity $v$. However, this capability can be considered an hypothesis at the moment: the validation of this theoretical approach needs in fact much more tests on many other different data sets.

4 Range values taken from Table 1, considering the "hospitalized data". 
Research funding: None declared.

Author contribution: All authors have accepted responsibility for the entire content of this manuscript and approved its submission.

Competing interests: Authors state no conflict of interest.

Informed consent: Not applicable.

Ethical approval: Not applicable.

\section{References}

Alonso, M., and E. J. Finn. 1983. Fundamental University Physics - Volume III, Quantum and Statistical Physics, 350. Addison-Wesley Publishing Company.

Becchetti, L., G. Conzo, P. Conzo and F. Salustri. 2020. "Understanding the Heterogeneity of Adverse COVID-19 Outcomes: the Role of Poor Quality of Air and Lockdown Decisions.” SSRN 3572548.

Biazar, J. 2006. "Solution of the Epidemic Model by Adomian Decomposition Method." Applied Mathematic and Computation 173: 1101-6.

Byrne, A. W., D. McEvoy, A. B. Collins, K. Hunt, M. Casey, A. Barber, F. Butler, J. Griffin, E. A. Lane, C. McAloon, K. O’Brien, P. Wall, K. A. Walsh, and S. J. More. 2020. "Inferred Duration of Infectious Period of SARS-CoV-2: Rapid Scoping Review and Analysis of Available Evidence Asymptomatic and Symptomatic Covid-19 Cases.” medRxiv. https://doi.org/10.1101/2020 .04.25.20079889.

Coker, E. S., L. Cavalli, and E. Fabrizi. 2020. "The Effects of Air Pollution on COVID-19 Related Mortality in Northern Italy." Environmental and Resource Economics 76: 611-34.

Cori, A., N. M. Ferguson, C. Fraser, and S. Cauchemez. 2013. "A New Framework and Software to Estimate Time-Varying Reproduction Numbers during Epidemics.” American Journal of Epidemiology 178 (9): 1505-12.

Davies, N. G., P. Klepac, Y. Liu, K. Prem, M. Jit, and R. M. Eggo, CMMID COVID-19 Working Group. 2020a. “Age-Dependent Effects in the Transmission and Control of Covid-19 Epidemics." Nature Medicine 26: 1205-11.

Davies, N. G., A. J. Kucharsky, R. M. Eggo, A. Gimma, W. J. Edmunds, and CMMID COVID-19 Working Group. 2020b. "The Effect of Non-Pharmaceutical Interventions on Covid-19 Cases, Deaths and Demand for Hospital Services in the UK: a Modelling Study." The Lancet Public Health 5 (7): E375-85.

Distante, C., P. Piscitelli, and A. Miani. 2020. "Covid-19 Outbreak Progression in Italian Regions: Approaching the Peak by March 29th.” MedRxiv preprint. https://doi.org/10.1101/2020.03.30.20043612.

DPCM 2020, Gazzetta Ufficiale Della Republica Italiana n. 59 Dell'8 Marzo 2020.

Du, Z., X. Xu, Y. Wu, L. Wang, and J. Benjamin. 2020. "Cowling, Lauren Arcel Meyers, Serial Interval of Covid-19 Among Publicly Reported Confirmed Cases.” Emerging Infectious Diseases 26 (N 6): 1341-3.

Gaeta, G. 2020. "Data Analysis for the Covid-19 Early Dynamics in Northern Italy." arXiv: 2003.02062v1 [q-bio.PE].

Gandhi, M., D. S. Yokoe, and D. V. Havlir. 2020. "Asymptomatic Transmission, the Achilles' Heel of Current Strategies to Control Covid-19 - Editorial.” New England Journal of Medicine 382 (22): 2158-60.

Goumenou, M., D. Sarigiannis, A. Tsatsakis, O. Anesti, A. O. Docea, D. Petrakis, D. Tsoukalas, R. Kostoff, V. Rakitskii, D. A. Spandidos, M. Aschner, and D. Calina. 2020. "Covid-19 in Northern Italy: An Integrative Overview of Factors Possibly Influencing the Sharp Increase of the Outbreak (Review).” Molecular Medicine Reports 22: 20-32.

Harkema, S., A. Behrman, and H. Barbeau. 2012. Handbook of Clinical Neurology - Chapter 16. Elsevier.

Harko, T., F. S. N. Lobo and M. K. Mak. 2012. "Exact Analytical Solution of the Susceptible-Infected-Recovered (SIR) Epidemic Model and the SIR Model with Equal Death and Birth Rates." 236: 184-94.

ISTAT 2013 Italia in Cifre.

Italian Department of Civil Protection. 2020. http://opendatadpc.maps.arcgis.com/apps/opsdashboard/index.html\#/ b0c68bce2cce478eaac82fe38d4138b1.

Italian Ministry of Health. 2020. Daily Bulletin Covid-19 Outbreak in Italy. http://www.salute.gov.it/imgs/C_17_pagineAree_ 5351_file.pdf.

Italian National Institute of Health (ISS). 2020. Coronavirus Surveillance Bulletin March 19/20. https://www.epicentro.iss.it/ coronavirus/bollettino/Bollettino\%20Sorveglianza\%20integrata\%20COVID-19_19-marzo202020.pdf.

Jones, N. R., Z. U. Qureshi, R. J. Temple, J. P. J. Larwood, T. Greenhalgh and L. Bourouiba. 2020. "Two Metres or One? What is the Evidence for Physical Distancing in Covid-19?.” British Medical Journal 370: m3223.

Kermack, W. O., and A. G. McKendrick. 1927. "Contribution to the Mathematical Theory of Epidemics." Proceedings of the Royal Society of London - Series A: Mathematical and Physical Sciences 115: 700-21.

Lauer, S. A., K. H. Grantz, Q. Bi, F. K. Jones, Q. Zheng, H. R. Meredith, A. S. Azman, N. G. Reich, and J. Lessler. 2020. “The Incubation Period of Coronavirus Disease 2019 (COVID-19) from Publicly Reported Confirmed Cases: Estimation and Application." Annals of Internal Medicine 172: 577-82. 
Li, Yi., M. Liang, X. Yin, X. Hao, H. Meng, Z. Hu, Y. Wang, and L. Jin. “Covid-19 Epidemic Outside China: 34 Founders and Exponential Growth.” MedRxiv preprint. https://doi.org/10.1101/2020.03.01.20029819.

Li, R., S. Pei, B. Chen, Y. Song, T. Zhang, W. Yang, and J. Shaman. 2020. "Substantial Undocumented Infection Facilitates the Rapid Dissemination of Novel Coronavirus (SARS-CoV2).” Science 368: 489-93.

Ma, S., J. Zhang, M. Zeng, Q. Yun, W. Guo, Y. Zheng, S. Zhao, M. H. Wang, and Z. Yang. 2020. "Epidemiological Parameters of Coronavirus Disease 2019: a Pooled Analysis of Publicly Reported Individual Data of 1155 Cases from Seven Countries." medRxiv. https://doi.org/10.1101/2020.03.21.20040329.

Pagano, A., E. V. Pagano, G. Pagano, and S. Spartà. 2020. “A Note on Corona-virus (Covid-19) Infection in Italy.” The European Physical Journal Plus 135: 667.

Rafei, M., H. Daniali, and D. D. Ganji. 2007a. "Variational Iteration Method for Solving the Epidemic Model and the Prey and Predator Problem." Applied Mathematics and Computation 186: 1701-9.

Rafei, M., D. D. Ganji, and H. Daniali. 2007b. "Solution of the Epidemic Model by the Homotopy Perturbation Method." Applied Mathematics and Computation 187: 1056-62.

Statista. 2020. https://www.statista.com/statistics/376531/walking-distance-in-england-uk-by-age-and-gender/ (accessed October 14, 2020).

Tuite, A. R., D. N. Fisman, and A. L. Greer. 2020. "Mathematical Modelling of Covid-19 Transmission and Mitigation Strategies in the Population of Ontario, Canada, CMAJ.” Canadian Medical Association Journal. 192: E497-505.

TWu, J., K. Leung, and G. M. Leung. 2020. "Nowcasting and Forecasting the Potential and International Spread of the 2019-nCoV Outbreak Originating in Wuhan, China: a Modelling Study." Lancet 395 (10225): 689-97.

Zhao, S., Q. Lin, J. Ran, S. Musa, G. Yang, W. Wang, Y. Lou, D. Gao, L. Yang, D. He, and M. Wang. 2020. “Preliminary Estimation of the Basic Reproduction Number of Novel Coronavirus (2019-nCoV) in China, from 2019 to 2020: a Data Driven Analysis in the Early Phase of the Outbreak." International Journal of Infectous Disease 92: 214-17.

Zhou, T., Q. Liu, Z. Yang, J. Liao, K. Yang, W. Bai, X. Lu, and W. Xang. 2020. "Preliminary Prediction of the Basic Reproduction Number of the Wuhan Novel Coronavirus 2019-nCoV.” Journal of Evidence-Based Medicine 13 (1): 3-7. 